by firms, and working in direct touch with the conditions and requirements of commercial development, can never be fully undertaken by other institutions or organisations. At the same time, metallurgists owe a great debt of gratitude to the universities. As an example, it may be mentioned that the training and research work carried out in the laboratories of the Applied Science Department of the University of Sheffield are worthy of the highest traditions of the city, and the establishment of metallurgy as an independent faculty with, quite recently, a special founding course and degrees in foundry science is but one instance of the way in which the authorities are keeping in the forefront of metallurgical progress. The importance of research facilities is equally recognised at other universities, each in its own sphere, but in regard to ferrous metallurgy the University of Sheffield naturally occupies a special position and is second to none in the world.

Outside the many and valuable researches carried out at the various universities of Great Britain reference must certainly be made to the important work being carried on continuously by the National Physical Laboratory, originally under the able guidance of Sir Richard Glazebrook, and now that of Sir Joseph Petavel, to whom and his staff, composed of men of scientific eminence, great credit is due. This great national establishment is now supported by large annual grants, aided by Parliament through the Royal Society and the Department of Scientific and Industrial Research.

\section{The Future}

Finally, as will have been gathered in the reading of this article, the continued and increasing use of alloy steels in their many forms is most certainly assured. The great value of such products can almost be said to be beyond computation in view of the services they render, and modern civilisation could not be sustained by any other means ; they may, therefore, be justly said to be beyond any adequate expression in terms of money.

It is scarcely realised how largely further advances of present modern civilisation depend upon alloy steels. Take these away and our modern civilisation could certainly not exist, at any rate, on anything like its present high plane, and we should in many respects be cast back to the comparatively unsatisfactory conditions of more than a century ago.

It is entirely the production and use of alloy steels which have rendered practicable the bringing into being of engineering and other constructions which would not otherwise have been possible, whether in their employment on land or sea or in the air.

One useful alloy after another, new or improved, takes its place in the eivilisation which it advances and thereafter it is indispensable unless indeed a still better material can be discovered. Progress far beyond the bounds which could be foreseen twenty-five years ago has already been achieved, and the rate of such advance appears likely to continue undiminished. Therein lie challenge, inspiration and encouragement for those of the younger generation.

\title{
Progress in Radio Communication
}

\section{By Prof. E. V. Appleton, f.R.S., Wheatstone Professor of Physics, King's College, London}

$\mathrm{T}^{\mathrm{T}}$ is a matter worthy of note that the remarkable progress of radio communication during the last quarter of a century has proceeded largely from the exploitation of the properties of that late. nineteenth century discovery, the free electron. For it is no exaggeration to say that practically all the instrumental progress of the period is connected in some way with that wonderful device, the three-electrode electron tube, while we now realise that the spectacular annihilation of distances in round-the-world communication by wireless is only brought about by the beneficent influence of free electrons at high atmospheric levels. Another significant feature of the period is that radio developments have had an unusually marked influence on human intercourse for, through broad. casting, there has been introduced a new and permanent feature of social and cultural enlightenment while, by way of the oversea wireless telephone, the different parts of the Empire have been brought into a closer unity which from time to time has been sealed by personal messages of greeting from His Majesty the King to all his subjects throughout the world.

In 1910 the utility of wireless communication was regarded as being largely concerned with increasing the safety of life at sea. Public interest was, in 1912 for example, stirred by the case of the Titanic which, with some 3,000 people on board, struck an iceberg on her maiden voyage 
across the Atlantic. Several ships picked up her wireless distress signals and raced to the rescue. Unfortunately, the Titanic had sunk when help arrived, and only 900 persons were saved, though it is clear that without the aid of wireless all would have been lost. The first of a series of international conferences concerning the safety of life at sea was held in the following year, though it was not until after the War that stringent regulations concerning the provision of wireless equipment for ships of above a specified tonnage were put into force.

Technicians were, at first, slow to recognise the potentialities of the instrument which was to revolutionise the subject, for it was not until 1913 that Armstrong, Meissner and Round independently discovered that the three-electrode valve, which de Forest had invented so far back as 1907, was capable of generating continuous electrical oscillations, and not until after the War was general use made of it in wireless telephony. Rather was attention being paid at that time to the possibilities of the Poulsen arc, and the different types of alternators designed by Goldschmidt, Alexanderson and others. The general tendency before the War was, in fact, to use arcs ant alternators for high-power sending stations ar $j$ spark generators for short-distance communication between ships.

Meanwhile, the fuller utilisation of the threeelectrode valve in reception (for detection and amplification) was limited by its inconstancy in operation, which was due to the imperfect vacuum then realisable. A notable improvement was effected in this direction by Langmuir who, in 1915, produced 'hard' valves from which all deleterious traces of gas had been removed. It thus came about that after the War there was to the hand of the experimenter a device, constant and reliable in operation, which, for sending, would produce sustained waves over a very wide range of lengths, and, on the receiving side, give, in cascade, magnifications of a million-fold. With such a generator, any form of information, whether concerning a sound or a picture, could be transmitted over a distance in the form of a modulation of the waves; while with such an amplifier in use at the receiving end of a wireless circuit, the power required at the sender could be correspond. ingly reduced.

In the summer of 1922 some of the leading British manufacturers of wireless apparatus approached the Post Office for permission to begin an experimental service of broadcasting speech and music by wireless telephony in this country, and the result was the formation of the British Broadcasting Company. Under the direction of this Company and its successor, the British Broadcasting Corporation, which on January 1, 1927, was incorporated by Royal Charter, there has grown up a great service of sound broadcasting in Great Britain, while to provide listeners with the necessary apparatus for reception there has arisen a new and vigorous electrical industry. In the evolution of the modern wireless receiver, commercial research has led to the development of new types of valve filaments giving greater efficiency and to more elaborate types of electrode structures. In this connexion special mention must be made of the immediate derivatives of the three-electrode valve, namely, the screen-grid valve for high-frequency amplification and the pentode for distortionless amplification of strong signals

Most histories contain a revolution, and the history of wireless communication is no exception to the general rule. At the end of the War, it was regarded as established that long waves were superior to short waves for long-distance communications. The superiority was, in fact, embodied in a transmission formula due to Austin, which was then much employed by radio engineers. It is generally thought that the first hint of the extraordinary possibilities of short waves for longdistance communication came from the experiments of amateurs in December 1921. Such experimenters were limited by law to use wavelengths less than 200 metres but, though operating with very small power indeed, they were able to establish contact with other amateurs 7,000 miles away. During the winter of $1922-23$, Dr. W. H. Eccles and H. Morris-Airby, using receivers tuned to wave-lengths lower than 100 metres, were able almost every night to pick up signals from American amateur stations, often as harmonics of the sending wave length. In October 1924 the greatest distance of all was spanned when communication was established between $\mathrm{F}$. Bell in New Zealand and C. W. Goyder, a Mill Hill schoolboy.

The revolution mentioned above came at a time when the problem of linking up the Empire by wireless was under discussion. In March 1923 the British Government announced the decision to erect a large wireless station at Rugby, capable of communicating with any part of the world. By the end of 1926 this station, working on long waves, as well as a wireless telephone long-wave link between Great Britain and North America, were in successful operation. The latter service, the details of which were worked out by the engineers of the British Post Office in collaboration with the engineers of the American Telegraph and Telephone Company, was the first long-distance 
commercial wireless-telephone link to be completed, and its inauguration constitutes a landmark in the history of electrical communication. In connexion with Empire telegraph communications by short waves the engineers of the Marconi Company did pioneer work. The most notable invention in this field is due to C. S. Franklin who showed how, by using a special array of aerial wires with a similar array behind, to act as a reflector, it was possible to confine the waves into a directed beam. Nowadays, for all point-to-point services, advantage is taken of such directional and economic projection of the waves.

To the short wave beam station built by the Marconi Company for telegraph communication to the Dominions, there have been added direct wireless telephone services to different parts of the Empire. In these cases also beam senders and receivers, built by the Post Office, are used, with corresponding stations oversea. The sending stations are all accommodated at Rugby, while the corresponding receiving stations are situated at Baldock. The result of these direct Empire services is that it is not an exaggeration to say that the whole of the Empire is 'on the telephone', with London as the exchange. A further experiment in Imperial communication has been the inauguration of Empire short-wave broadcasting by the B.B.C. in 1932. At first it was attempted to provide a two-hours' programme in each of five Empire zones between the convenient hours of 6 p.m. and midnight, local time, while the B.B.C. publication, World Radio, which is the official organ of Empire broadcasting, is published so far in advance that copies of it are available in different parts of the Empire at the appropriate time.

On the more purely scientific side, substantial progress has been made during the last twentyfive years, especially as regards the elucidation of electric wave propagation. For the propagation of waves along the ground the correctness of the transmission formula of Sommerfeld has been checked by signal intensity measurements made by Barnett and Ratcliffe and by B.B.C. engineers. Other quantitative experiments have been carried out to elucidate the structure and properties of the highly ionised region of the upper atmosphere (from $100 \mathrm{~km}$. upwards) known as the ionosphere, which reflects wireless waves and makes longdistance radio communication possible. The existence of such a reflecting stratum was postulated so far back as 1905 by both Kennelly and Heaviside, but it was not until 1924 that its situation in the atmosphere was established. Further investigations have shown that above the KennellyHeaviside layer, as the region about $100 \mathrm{~km}$. is called, there is a more intensely ionised stratum, the electrical density of which is the factor that limits the shortest wave-length which can be used for round-the-world communication. Experiments carried out by Henderson, Rose and others during the solar eclipse of 1932 in Canada have shown that the upper atmospheric ionisation is caused by the photo-electric action of sunlight. Due to the influence of the earth's magnetic field on the motion of the free electrons, the ionosphere is a doubly-refracting medium and resolves an incident wireless wave into two oppositely rotating components which experience absorption of different amounts. The result is that a reflected wireless wave of, say, 400 metres, is of predominantly left-handed polarisation in the northern hemisphere but of opposite rotation in the southern hemisphere.

The ionisation in the upper atmosphere is not by any means constant. It is usually denser by day than by night and denser in summer than in winter, but these normal variations are sometimes profoundly upset by manifestations of solar activity. The general effect of such activity has been found to be an alteration of the disposition of the electrification and thus of the properties of the ionosphere as a reflecting medium. One of the curious things noticed, in our latitudes, is the difference in the effects on long-wave and on shortwave transmission. With long waves a solar disturbance which produces a magnetic storm may often increase the daylight signal intensity, but in the case of short wave channels there is almost always a sharp reduction in signal strength. In the case of communication over a great circle which traverses high latitudes, the effects of a magnetic storm have been found to be specially marked, which explains why the short wave channels to Canada are more frequently interrupted in this way than are the services to South Africa.

On the purely technical side there have been, particularly since the War, many notable contributions to electrical circuit practice, quite apart from the thermionic valve and its derivatives. Selection is difficult, but perhaps the superheterodyne circuit of Armstrong and the quartz crystal oscillator of Cady may be chosen as outstanding. The superheterodyne receiver, in which amplification is effected after the conversion of the received signals to a lower frequency, is almost universally used nowadays in commercial practice and in the more elaborate broadcast receivers; while the quartz oscillator, particularly in the hands of Dye and his associates at the National Physical Laboratory, has been developed to provide high-frequency standards of a constancy and accuracy hitherto unattainable. 